\title{
The burden of clostridium difficile infection: estimates of the incidence of CDI from U.S. Administrative databases
}

Margaret A. Olsen ${ }^{1,2^{*}}$, Yinong Young-X ${ }^{3}$, Dustin Stwalley ${ }^{1}$, Ciarán P. Kelly ${ }^{4}$, Dale N. Gerding ${ }^{5}$, Mohammed J. Saeed', Cedric Mahé ${ }^{1}$ and Erik R. Dubberke ${ }^{1}$

\begin{abstract}
Background: Many administrative data sources are available to study the epidemiology of infectious diseases, including Clostridium difficile infection (CDI), but few publications have compared CDI event rates across databases using similar methodology. We used comparable methods with multiple administrative databases to compare the incidence of CDI in older and younger persons in the United States.

Methods: We performed a retrospective study using three longitudinal data sources (Medicare, Optumlnsight LabRx, and Healthcare Cost and Utilization Project State Inpatient Database (SID)), and two hospital encounter-level data sources (Nationwide Inpatient Sample (NIS) and Premier Perspective database) to identify CDI in adults aged 18 and older with calculation of CDI incidence rates/100,000 person-years of observation (pyo) and CDI categorization (onset and association).

Results: The incidence of CDI ranged from 66/100,000 in persons under 65 years (LabRx), 383/100,000 in elderly persons (SID), and 677/100,000 in elderly persons (Medicare). Ninety percent of CDI episodes in the LabRx population were characterized as community-onset compared to $41 \%$ in the Medicare population. The majority of CDI episodes in the Medicare and LabRx databases were identified based on only a CDI diagnosis, whereas almost $3 / 4$ of encounters coded for CDI in the Premier hospital data were confirmed with a positive test result plus treatment with metronidazole or oral vancomycin. Using only the Medicare inpatient data to calculate encounter-level CDI events resulted in 553 CDI events/100,000 persons, virtually the same as the encounter proportion calculated using the NIS (544/100,000 persons).

Conclusions: We found that the incidence of CDI was $35 \%$ higher in the Medicare data and fewer episodes were attributed to hospital acquisition when all medical claims were used to identify CDI, compared to only inpatient data lacking information on diagnosis and treatment in the outpatient setting. The incidence of CDI was 10-fold lower and the proportion of community-onset CDI was much higher in the privately insured younger LabRx population compared to the elderly Medicare population. The methods we developed to identify incident CDI can be used by other investigators to study the incidence of other infectious diseases and adverse events using large generalizable administrative datasets.
\end{abstract}

Keywords: Clostridium difficile, Incidence, Administrative data, Claims data, Medicare data, Hospital-acquired infection

\footnotetext{
* Correspondence: molsen@wustl.edu

'Division of Infectious Diseases, Washington University School of Medicine,

Campus Box 8051660 S. Euclid Ave, St. Louis 63110MO, USA

${ }^{2}$ Department of Surgery, Washington University School of Medicine, St. Louis,

MO, USA

Full list of author information is available at the end of the article
} 


\section{Background}

Clostridium difficile infection (CDI) incidence in the United States has increased dramatically since 2000 $[1,2]$. The number of discharges from non-federal, acute care hospitals assigned the International Classification of Diseases, Ninth Revision, Clinical Modification (ICD-9-CM) diagnosis code for CDI (008.45) increased by 2.7 -fold between 2000 and 2012 using data from the Healthcare Cost and Utilization Project (HCUP) Nationwide Inpatient Sample (NIS) [3]. CDI was estimated to cause as many as 14,000 deaths in 2007 and an attributable mortality ranging from $5.7 \%$ in endemic settings to $16.7 \%$ in severe outbreaks since 2000 [2, 4-7].

Much research has focused on identifying specific risk factors for CDI, but this might not be the best approach to identify high risk populations. The results of risk factor studies have not always been consistent [8-15], with potential reasons for discrepancies including differences in patient populations, data availability, and/or study definitions. These differences limit both the ability to compare results across studies and the generalizability of results, making it difficult to identify which populations have the highest CDI burden and how to best target CDI prevention practices.

Billing and claims data (referred to collectively as administrative data) are increasingly used for health services and outcomes research because of large population sizes, generalizability of findings, and the ability to follow individuals across the spectrum of health care. Unfortunately there is no single, comprehensive database in the U.S. that can be used to identify all populations at risk for CDI. In order to better understand the epidemiology of CDI we applied common definitions to identify and classify CDI from five large administrative databases, the Medicare $5 \%$ Sample, HCUP State Inpatient Databases (SID) and the NIS, OptumInsight ${ }^{\mathrm{tm}}$ Retrospective Database (LabRx), and Premier Perspective, to improve our understanding of the burden of CDI in the U.S. from a population perspective.

\section{Methods}

The databases used for this study were anonymized; some contained encrypted identifiers to link longitudinal data within a person ("cohort" data), while the others consisted of only unlinked hospital billing data. The hospital billing databases (NIS, Premier) were analyzed at the hospital discharge level. The cohort databases containing a person-level identifier to track persons across healthcare encounters (Medicare, LabRx, and SID) were analyzed at both the person-level and hospital dischargelevel. For all cohort data hospitalizations with same-day transfers to the same or a different hospital were aggregated and treated as a single hospital stay, to avoid over- counting long hospitalizations or direct transfers as distinct hospital visits. The Washington University Human Research Protection Office and Geisel School of Medicine at Dartmouth Committee for the Protection of Human Subjects gave approval to conduct this research with a waiver of informed consent.

\section{Identification of CDI}

Criteria used to identify CDI combined any of the following:

1) ICD-9-CM diagnosis code for CDI (008.45) during an inpatient hospital stay;

2) ICD-9-CM diagnosis code for CDI in an outpatient encounter with specific restrictions (see Additional file 1: Appendix);

3) Positive test result for $C$. difficile toxins or toxin genes (LabRx); and

4) Non-topical metronidazole or oral vancomycin therapy within \pm 14 days of a CPT- 4 code for a C. difficile test or diagnosis code for CDI (Medicare, LabRx, and Premier).

For person-level analyses, subsequent unique episodes of CDI were identified if the person met criteria for CDI again after an 84 day period during which there were no healthcare encounters meeting the CDI case definition. We used a conservative definition for subsequent unique episodes of CDI to minimize misclassifying carry-forward of the CDI diagnosis code or CDI recurrence as a unique episode of CDI.

\section{Inclusion/exclusion criteria}

For Medicare and LabRx data, enrollment and complete health insurance coverage for the year prior to the first onset date of CDI was required. For Medicare age $\geq$ 66 years at the time of CDI onset was required; for the SID, NIS, and Premier all persons aged $\geq 18$ years were included. Individuals 65 and older were excluded from the LabRx data since they represented only $7 \%$ of the privately insured population. For the cohort data CDI episodes were excluded if the person had CDI within the prior 84 days in order to identify new episodes of CDI in 2009.

\section{Date of onset and determination of the location of onset and attribution of $\mathrm{CDI}$}

The date of onset of CDI was defined as the first date corresponding to a coded diagnosis of CDI. In the LabRx data, if a CDI toxin test was performed, the date of the first positive test was used as the date of CDI onset.

The location of onset and attribution for each CDI episode was determined using an algorithm based on the most recent SHEA/IDSA definitions [16-18]. CDI coded 
during a hospitalization was classified as communityonset if: 1) CDI was the primary diagnosis; 2) the primary diagnosis was diarrhea, abdominal pain, or nausea and CDI was coded in a secondary position; or 3) CDI was coded in a secondary position and the hospital length of stay was $\leq 3$ days. If no further information was available from outpatient or physician claims, CDI was classified as hospital-onset if it was coded in a secondary position and the hospital length of stay was $>3$ days. If the database did not contain a common person identifier, no further categorization beyond community- or hospitalonset was possible. If a common person identifier was available and the CDI episode was community-onset, hospitalizations and other healthcare facility exposures prior to the CDI hospital admission were identified to classify the episode (see Additional file 1: Appendix).

\section{Analysis}

The rate of CDI in a population group was defined as the number of CDI episodes divided by the person-years of observation (pyo, defined from $1 / 1 / 2009$ up to the next CDI event, death, or $12 / 31 / 2009$, whichever came first). For the SID the population of adults aged 18-64 and the elderly in the seven states was obtained from the 2010 census (www.census.gov). Person-years in the
SID data were calculated taking into account death (using the midpoint of the death discharge quarter to define the date of death). SAS version 9.3 and SPSS 20.0 were used for data management and analysis.

\section{Results}

The demographic characteristics and number of hospitalizations and outpatient encounters in the different databases are shown in the Additional file 1: Appendix. In the three longitudinal datasets approximately $0.2 \%$ of the initially identified hospitalizations coded for CDI were excluded because the patient was previously identified with CDI within the prior 12 weeks. The criteria used to identify CDI are shown in Table 1 . In the Medicare data $23 \%$ of inpatient CDI episodes were identified by the CDI diagnosis code together with an outpatient prescription for metronidazole or oral vancomycin within 14 days after hospital discharge; when restricted to patients with Part D coverage this corresponded to $40 \%$ of inpatient CDI episodes (1303/3280). In the Medicare data approximately $53 \%$ of unique CDI inpatient hospital episodes were identified by a secondary diagnosis code during the hospitalization, consistent with hospital-onset CDI, compared to $70 \%$ in the SID, and $23 \%$ in the LabRx data. In the encounter-level

Table 1 CDI Episodes in 2009 according to the definition used to identify CDI

\begin{tabular}{|c|c|c|c|c|c|}
\hline Definition & Medicare $^{a}$ & $\mathrm{Lab} / \mathrm{Rx}^{\mathrm{a}}$ & $\mathrm{SID}^{\mathrm{a}}$ & Premier & NIS \\
\hline Positive test + diagnosis $+\mathrm{Rx}$ & N/A & N/A & - & $4,623(72.8)$ & - \\
\hline $\begin{array}{l}\text { Diagnosis }+ \text { Rx within } 14 \text { days after hospital discharge } \\
\text { or during hospitalization }\end{array}$ & $1,303(23.4)$ & $1,088(65.5)$ & - & $1,347(21.2)$ & - \\
\hline Diagnosis + Positive test during hospitalization & N/A & N/A & - & $108(1.7)$ & - \\
\hline Primary diagnosis & $994(17.8)$ & $194(11.7)$ & $20,894(30.5)$ & $13(0.02)$ & $110,553(32.8)$ \\
\hline Diagnosis on admission & $338(6.1)$ & N/A & N/A & $2(<0.01)$ & N/A \\
\hline Positive test only & N/A & N/A & N/A & $205(3.2)$ & N/A \\
\hline Secondary diagnosis & $2,941(52.7)$ & $379(22.8)$ & $47,546(69.5)$ & $52(0.08)$ & $226,012(67.2)$ \\
\hline Total Inpatient CDI & 5,576 & 1,661 & 68,440 & 6,350 & 336,565 \\
\hline $\begin{array}{l}\text { Positive test }+ \text { Diagnosis }+R x \text { within }+/-14 \text { days } \\
\text { of diagnosis or positive test date(s) }\end{array}$ & N/A & $87(2.7)$ & - & - & - \\
\hline $\begin{array}{l}\text { Diagnosis on } \geq 2 \text { service dates }+R x \text { within }+/-14 \text { days } \\
\text { of diagnosis date(s) }\end{array}$ & $422(10.4)$ & $409(12.6)$ & - & - & - \\
\hline Positive test + diagnosis within $+/-14$ days & N/A & $6(0.2)$ & - & - & - \\
\hline Positive test $+R x$ within $+/-14$ days & N/A & $436(13.4)$ & - & - & - \\
\hline $\begin{array}{l}\text { Diagnosis }+R x+\text { lab test within }+/-14 \text { days of the diagnosis } \\
\text { or lab test date(s) }\end{array}$ & $133(3.3)$ & $148(4.6)$ & - & - & - \\
\hline Diagnosis + Rx within $+/-14$ days & $334(8.2)$ & $524(16.1)$ & - & - & - \\
\hline Positive test only & N/A & $262(8.1)$ & - & - & - \\
\hline Diagnosis only & $3,162(77.6)$ & $1,251(38.5)$ & - & - & - \\
\hline CDI test $+R x$ & $25(0.6)$ & $129(4.0)$ & - & - & - \\
\hline Total Outpatient CDI & 4,076 & 3,252 & - & - & - \\
\hline
\end{tabular}

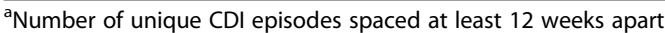

$\mathrm{Rx}=$ non-topical metronidazole or oral vancomycin therapy 
Premier data, 73 \% of CDI hospitalizations were identified by a $C$. difficile laboratory test, diagnosis, plus metronidazole or oral vancomycin therapy, and $21 \%$ were identified based on the CDI diagnosis code plus treatment without a positive test result.

Approximately $42 \%$ of the CDI episodes in the Medicare data were first identified in the outpatient setting (Table 1). Of these outpatient CDI episodes, $78 \%$ were identified by the CDI diagnosis code alone, and $21.8 \%$ were identified by the diagnosis code plus outpatient CDI prescription (35\% for individuals with Part D coverage). Fifteen percent (610/4076) of the persons identified with CDI outside of the hospital were hospitalized within 14 days of CDI diagnosis; of these $60 \%(364 / 610)$ were coded for CDI during the inpatient hospitalization. In the LabRx data of younger persons, $38.5 \%$ of outpatient CDI episodes were identified by the CDI diagnosis code only with no supporting laboratory or prescription evidence for infection. A total of $28.7 \%$ of the outpatient CDI episodes in the LabRx data were identified by a diagnosis code plus therapy, while $13.4 \%$ of outpatient CDI episodes were identified by a positive C. difficile test plus therapy within 14 days.

The categorization of CDI episodes by database is shown in Table 2. Fifty-nine percent of CDI episodes (5648/9652) were categorized as healthcare facility onset (hospital or other facility) in the Medicare data, compared to $68 \%$ in the SID $(46,739 / 68,440)$. Communityonset healthcare facility-associated CDI made up $13 \%$ of the CDI episodes in Medicare, compared to $11.8 \%$ in the SID. Community-onset community-associated CDI episodes included $22.6 \%$ of episodes in Medicare vs. $13.9 \%$ in the SID and $35.2 \%$ in the Premier data. Only $22.4 \%(1102 / 4913)$ of the CDI episodes in the LabRx data were healthcare facility associated (excluding indeterminate association), while $68.4 \%$ of episodes were categorized as community-onset community-associated.

The number of persons with one or more than one unique episode of CDI in the longitudinal datasets is shown in Table 3 and the cumulative incidence of CDI in Table 4. $2.6 \%$ of persons in the Medicare and
Table 3 Number of persons with multiple incident CDI episodes (no other CDI diagnosis within 12 weeks)

\begin{tabular}{llll}
\hline & Medicare & LabRx & SID \\
\hline Size of the cohort & $1,465,927$ & $7,255,708$ & $5,575,935$ \\
No CDI & $1,456,526$ & $7,251,046$ & $5,508,903$ \\
Only one CDI & $9,155(97.4)^{\mathrm{a}}$ & $4,429(95.0)$ & $65,549(97.9)$ \\
2 CDI & $241(2.6)$ & $215(4.6)$ & $1,403(2.1)$ \\
$>2$ CDI & $5(0.05)$ & $18(0.4)$ & $28(0.04)$ \\
\hline
\end{tabular}

${ }^{\mathrm{a}}$ Percentages refer to percentage of persons with $\mathrm{CDI}$

$5.0 \%$ of persons in the LabRx data had $>1$ unique episode of CDI spaced at least 12 weeks apart in 2009. The rate of CDI in the Medicare data was 677/ 100,000 pyo, while the rate was $43 \%$ lower (383) in the SID. The rate of CDI in the younger adult population in the SID was ten-fold lower (37.5) than the rate in the elderly SID population, while the rate of CDI in the LabRx data including outpatient CDI was 1.8-fold higher than in the SID younger population. The rate of hospital onset CDI per 10,000 patient days was higher in the SID for elderly persons (15.9) compared to the Medicare data (9.8), lower in the SID and Premier data for younger adults, and lowest (1.1) in the LabRx data.

To determine the impact of including outpatient medical claims and linkage within a person on CDI incidence, we compared the cumulative incidence, categorization of episodes, and attribution of CDI in the Medicare data when complete claims were used vs. only inpatient facility claims, with and without linkage within a person. When only the inpatient facility claims were used (analogous to the SID), the total number of CDI episodes was reduced to 6276 and the cumulative incidence of CDI decreased to 440/100,000 pyo. In addition, the number of hospital-onset cases and the rate of hospital-onset CDI increased while community-associated CDI decreased over two-fold (Table 5). When the person-level linkage in the inpatient Medicare data was removed (analogous to the

Table 2 Categorization of CDI episodes in the different databases

\begin{tabular}{|c|c|c|c|c|c|}
\hline & Medicare & $\mathrm{Lab} / \mathrm{Rx}$ & SID & Premier $^{a}$ & NIS \\
\hline Hospital-Onset & $3,223(33.4)$ & $449(9.1)$ & $46,347(67.7)$ & $3,984(64.8)$ & $211,344(62.8)$ \\
\hline Other Healthcare-Facility Onset & $2,425(25.1)$ & $54(1.1)$ & $392(5.7)$ & - & - \\
\hline Community-Onset Hospital-associated & $1,258(13.0)$ & $599(12.2)$ & $8,048(11.8)$ & - & - \\
\hline Community-Onset Indeterminate-association & $561(5.8)$ & $451(9.2)$ & $4,139(6.0)$ & - & - \\
\hline Community-Onset Community-associated & $2,185(22.6)$ & $3,360(68.4)$ & $9,514(13.9)$ & - & - \\
\hline Community-Onset Unknown association ${ }^{\mathrm{b}}$ & - & - & - & $2,161(35.2)$ & $125,221(37.2)$ \\
\hline
\end{tabular}

${ }^{\mathrm{a}}$ The number of CDI events is reduced by 205 due to diagnosis of CDI by a positive test result only

${ }^{b}$ Episodes in the Premier and NIS encounter-level databases were classified as community-onset unknown association since it was not possible to determine prior health care exposures due to lack of a person-level identifier 
Table 4 Burden of CDI in the elderly in 2009 in the different databases, including all episodes of CDI

\begin{tabular}{|c|c|c|c|c|}
\hline Adults $<65$ Years & Lab/Rx & SID & Premier & NIS \\
\hline Rate of CDI/100,000 person-years & 66.0 & $37.5^{\mathrm{a}}$ & N/A & N/A \\
\hline Rate of hospital onset CDI/10,000 pt. days & 1.1 & 5.7 & 5.4 & 6.9 \\
\hline Prevalence of CDI at admission/1,000 hospitalizations & N/A & 1.5 & 1.9 & 2.0 \\
\hline Rate of healthcare facility-associated CDI/10,000 pt. days & 2.1 & N/A & N/A & N/A \\
\hline Elderly & Medicare & SID & Premier & NIS \\
\hline Rate of CDI/100,000 person-years & 677 & $383^{b}$ & N/A & N/A \\
\hline Rate of hospital onset CDI/10,000 pt. days & 9.8 & 15.9 & 11.6 & 15.5 \\
\hline Prevalence of CDI at admission/1,000 hospitalizations & 5.4 & 4.7 & 6.3 & 6.2 \\
\hline Rate of healthcare facility-associated CDI/10,000 pt. days & 12.5 & N/A & N/A & N/A \\
\hline
\end{tabular}

are/100,000 persons in 7 states aged $18-64$ years

${ }^{b}$ rate/ 100,000 persons in 7 states aged $>=65$ years

NIS), the number of CDI episodes increased by almost $30 \%$ compared to the linked inpatient Medicare data (8108 vs. 6276), because of the inability to exclude hospitalizations coded for CDI in the prior 12 weeks. The number of CDI events/100,000 hospitalizations was $553 / 100,000$ hospitalizations using the unlinked data. When the 2009 NIS data was restricted to hospitalizations in elderly persons aged 65 years and older, the CDI hospitalization proportion was 544 CDI visits/100,000 hospitalizations.

\section{Discussion}

We used five types of billing or claims data to define the burden of CDI in U.S. adults in 2009. To our knowledge this is the first study to compare the burden of CDI from a population perspective in different administrative

Table 5 Comparison of the number of CDI episodes in 2009 in the Medicare data according to the extent of information used to identify CDI

\begin{tabular}{|c|c|c|c|c|}
\hline \multirow[b]{2}{*}{ Type of CDI } & \multirow[b]{2}{*}{ Definition of CDI } & \multicolumn{3}{|c|}{ Source of information from medicare files } \\
\hline & & Complete inpatient + outpatient & $\begin{array}{l}\text { Inpatient only } \\
\text { (comparable to SID) }\end{array}$ & $\begin{array}{l}\text { Inpatient-unlinked } \\
\text { (comparable to NIS) }\end{array}$ \\
\hline \# hospital stays & - & 488,344 & 488,344 & 522,921 \\
\hline Total Inpatient CDI episodes & - & 5,576 & 6,276 & 8,108 \\
\hline \multirow[t]{3}{*}{ Inpatient CDI Episodes } & Primary discharge diagnosis) & 1,464 & 1,690 & 2,084 \\
\hline & Admission diagnosis & 487 & 584 & 824 \\
\hline & Secondary discharge diagnosis & 3,625 & 4,002 & 5,200 \\
\hline Outpatient CDI episodes & - & 4,076 & N/A & N/A \\
\hline Total CDI episodes & - & 9,652 & 6,276 & 8,108 \\
\hline \multicolumn{5}{|c|}{ Categorization of CDI Episodes (\% of episodes) } \\
\hline Hospital-Onset & & $3,223(33.4)$ & $3,765(60.0)$ & $4,769(58.8)$ \\
\hline Other Healthcare Facility-Ons & & $2,425(25.1)$ & N/A & N/A \\
\hline Community-Onset Healthcar & Facility-associated & $1,258(13.0)$ & $938(14.9)$ & N/A \\
\hline Community-associated CDI & & $2,185(22.6)$ & $1,032(16.4)$ & $3,339(41.2)$ \\
\hline Indeterminate & & $561(5.8)$ & $541(8.6)$ & N/A \\
\hline \multicolumn{5}{|l|}{ Rate of CDI Episodes } \\
\hline \multicolumn{2}{|c|}{ Rate of CDI/100,000 person-years } & 677 & 440 & $553^{\mathrm{a}}$ \\
\hline \multicolumn{2}{|c|}{ Rate of hospital onset CDI/10,000 pt. days } & 9.8 & 11.4 & 14.1 \\
\hline \multicolumn{2}{|c|}{ Prevalence of CDI at admission/1,000 hospitalizations } & 5.4 & 5.3 & 6.5 \\
\hline \multicolumn{2}{|c|}{ Rate of healthcare facility-associated CDI/10,000 pt. days } & 12.5 & 14.2 & 14.1 \\
\hline
\end{tabular}


databases using standardized methods to identify and classify CDI. We used all available information to identify CDI, including outpatient prescription claims for metronidazole and vancomycin in the Medicare (Part D) and LabRx data, inpatient treatment in Premier, and outpatient $C$. difficile test results in LabRx.

Not surprisingly, we found a higher cumulative incidence of CDI in the databases that contained inpatient and outpatient data compared to only inpatient billing data, similar to what was reported recently using Kaiser Permanente data [19]. The number of CDI episodes per 100,000 elderly persons was almost 1.8 -fold higher in the Medicare data compared to the inpatient only longitudinal-SID. However, when only inpatient data were used to identify CDI in the Medicare population and the analysis was conducted at the person-level, the cumulative incidence of CDI was very close to that calculated in the SID (440 vs. $383 / 100,000$ pyo). The $54 \%$ increase in the cumulative incidence using complete (677) vs. inpatient-only Medicare data (440) emphasizes the importance of using complete data from inpatient and outpatient settings to calculate CDI incidence. In addition, when we treated the inpatient Medicare data as encounter-level (i.e., hospitalizations as unique encounters), the number of CDI events/100,000 hospitalizations was remarkably similar to the $544 / 100,000$ hospitalizations in elderly persons in the 2009 NIS.

More CDI cases were identified as hospital-onset in the datasets with only inpatient facility data, resulting in a higher apparent hospital-onset CDI incidence. The rate of hospital-onset CDI increased in the Medicare data to 11.4 cases/10,000 patient days when analysis was restricted to only inpatient facility claims, and this rate increased further when the linkage within a patient was ignored (14.1 cases/10,000 patient days). This suggests that analysis of encounter-level data, such as the NIS, may result in over-estimation of CDI hospital rates by as much as $25 \%$ due to continued coding in subsequent hospitalizations that are part of the same CDI episode, and that caution should be used when using these data for surveillance purposes.

In analysis of complete Medicare claims, $33 \%$ of the CDI events were categorized as hospital-onset, whereas in the analyses using only inpatient Medicare facility data, approximately $60 \%$ of the CDI events were categorized as hospital-onset, suggesting that hospital-onset cases will be over-estimated by almost two-fold when only inpatient claims or billing data are used. These results are consistent with previous reports of the overattribution of hospital-onset CDI [20,21] and the overestimation of CDI cases identified by the ICD-9-CM diagnosis code compared to positive $C$. difficile toxin assay from facility billing data $[20,22-25]$. More hospital-onset cases were identified in the HCUP and
Premier data, likely due to misclassification of CDI with onset in the community.

The addition of laboratory results in the LabRx data suggests that $20 \%$ of CDI episodes may be missed when analyzing data without $C$. difficile test results. Identification of fecal transplant in administrative data via CPT-4 and HCPCS codes (available beginning 2013) may aid in the identification of CDI in future, particularly in combination with a positive $C$. difficile test result. Interestingly, approximately three-quarters of the outpatient CDI episodes in the LabRx data were not supported by a positive test result, with $38 \%$ identified on the basis of a CDI diagnosis alone. The percentage of outpatient CDI diagnoses without confirmation by a positive $C$. difficile laboratory test was very similar in our previous study using Veterans Administration data, in which only $32 \%$ of the total outpatient CDI cases had a $C$. difficile test result [26]. Further studies to validate the use of the ICD-9-CM diagnosis code for CDI in the outpatient setting in the absence of positive $C$. difficile test results are warranted to determine the accuracy of coding outside of the hospital.

In the Medicare data $23 \%$ of CDI episodes first diagnosed during an inpatient hospital stay were linked to a filled outpatient prescription consistent with CDI treatment within 2 weeks after hospital discharge. Since $47.5 \%$ of the Medicare patients had Part D coverage, this would suggest that almost half of elderly persons diagnosed with CDI during an inpatient hospitalization continue CDI treatment after leaving the hospital. In the LabRx data almost two-thirds of episodes identified during a hospitalization were linked to outpatient CDI treatment. We identified temporally related treatment of outpatient CDI in at most one-half of persons with prescription drug coverage in the Medicare (46\%) and LabRx (49 \%) data. In contrast, in the Premier data containing inpatient medications, $73 \%$ of inpatient CDI episodes had evidence of treatment during the hospitalization. Despite lack of documentation of treatment for many CDI cases, particularly in the Medicare data, the overall incidence of CDI in the elderly of $677 / 100,00$ pyo is remarkably similar to the incidence of $628 / 1000,000$ elderly persons reported by the Centers for Disease Control and Prevention's Emerging Infections Program (EIP) for 2011 [2].

Lessa reported that $53 \%$ of CDI events $(159,700$ community-associated $+81,300$ community-onset, health care facility associated) in persons of all ages were community-onset using the EIP data [2], similar to the $41.4 \%$ community-onset CDI episodes we identified in the Medicare data. In the recent publication using Kaiser Permanente data, $76 \%$ of the CDI events were community-onset, with a total of $40 \%$ characterized as community-onset community-associated [19]. This is 
lower than our finding that almost $90 \%$ of the CDI events in the LabRx data from younger persons had onset in the community, with $68 \%$ characterized as community-onset community associated CDI. The varying proportions of CDI with onset outside of the hospital in the Medicare and LabRx data compared to the EIP data may be related to differences in age of the populations. In the EIP data, $44 \%$ of persons with CDI were $<65$ years of age, and the proportion of CDI that is community-associated CDI is higher in younger populations [27]. Consistent with our current study, $52 \%$ of laboratory-identified CDI during inpatient hospitalizations in the EIP were present at admission to the hospital [28].

\section{Conclusions}

The similarities between our findings concerning the incidence and site of onset of CDI from several different administrative databases with recent EIP results validate use of these administrative databases to identify populations at risk for CDI. We determined how results may be skewed when important information is missing, such as outpatient data and encrypted identifiers, and the advantages of using complete claims data allowing for substantiation of the CDI diagnosis using laboratory claims for CDI testing, pharmacy claims for CDI treatment, and other diagnoses consistent with CDI (e.g., diarrhea). Although there are limitations to use of administrative data, these databases offer the opportunity to analyze CDI from a population perspective, including data from many different hospitals and from other healthcare facilities. These databases can provide more complete information on the epidemiology of CDI and enrich our understanding of the impact of CDI on young and older persons in the U.S. In addition, the methods we developed to extract comparable information can be used to determine the incidence of other infectious diseases (e.g., MRSA, septicemia) and adverse events (e.g., deep venous thrombosis) in varying populations using a combination of different administrative databases.

\section{Ethics approval and consent to participate}

The Washington University Human Research Protection Office and Geisel School of Medicine at Dartmouth Committee for the Protection of Human Subjects gave approval to conduct this research with a waiver of informed consent.

\section{Consent for publication}

Not Applicable.

\section{Availability of data and materials}

Five different data sources were used for this study. None of the data sources can be shared by the authors, per data use agreements with the individual organizations. All of the data sources are available for purchase, as described below.

Medicare claims (Chronic Condition Warehouse $5 \%$ random sample), obtained from the Centers for Medicare and Medicaid Services and the Research Data Assistance Center (www.resdac.org).

OptumInsight $^{\text {tw }}$ Retrospective Database (formerly i3 Ingenix/LabRx), obtained from OptumInsight (www. optum.com).

Healthcare Cost and Utilization Project Nationwide Inpatient Sample (NIS) and State Inpatient Databases (SID), obtained from the Agency for Healthcare Research and Quality (www.hcup-us.ahrq.gov/databases.jsp).

Premier Perspective database, obtained from Premier, Inc (www.premierinc.com).

\section{Additional file}

Additional file 1: Appendix. Description of Databases. Appendix 1 includes descriptions of the cohort and hospital billing databases used in the study. Appendix 2. Definition and characterization of Clostridium difficile infection. Appendix 2 includes study inclusion and exclusion criteria and identification and classification of CDI. Appendix 3. Information used from Different Databases to Identify CDI. Table includes information available in the different databases that was used to identify CDI. Appendix 4. Comparison of Number of Persons and Encounters in the Different Databases. Table includes the initial number of persons, hospitalizations, and outpatient visits identified in the five databases and the final numbers after applying exclusion criteria. Appendix 5. Demographics of Populations from the Different Databases. Table includes the demographics of persons or hospital encounters in the five databases. (DOCX $25 \mathrm{~kb}$ )

\section{Abbreviations}

CCW: Chronic Condition Warehouse; CDI: Clostridium difficile infection; CMS: Centers for Medicare and Medicaid Services; CPT-4: Current Procedural Terminology, $4^{\text {th }}$ edition; EIP: Emerging Infections Program; HCUP: Healthcare Cost and Utilization Project; ICD-9-CM: International Classification of Diseases, Ninth Revision, Clinical Modification; LabRx: Optumlnsight LabRx data; MRSA: methicillin-resistant Staphylococcus aureus; NIS: Nationwide Inpatient Sample; pyo: person-years of observation; SID: State Inpatient Database.

\section{Competing interests}

Dr. Olsen reports personal fees from Sanofi Pasteur, Merck, and Pfizer, and grants from Cubist Pharmaceuticals and Pfizer outside the submitted work; Dr. Young-Xu reports personal fees from Sanofi-Pasteur outside the submitted work;

Mr. Stwalley reports other from Abbott Laboratories and Bristol-Myers Squibb outside the submitted work;

Dr. Kelly reports personal fees from Astellas, Cubist, Optimer, Novartis, Medlmmune, Merck, grants and personal fees from Sanofi-Pasteur, grants and personal fees from Optimer, grants from CSL-Behring, grants from Merck, and personal fees from QuantiaMed outside the submitted work; Dr. Kelly has a patent for Passive immunotherapy for CDI using IgA pending; Dr. Gerding holds patents for the treatment and prevention of CDI licensed to ViroPharma/Shire, is a consultant for Merck, Shire, Cubist, Rebiotix, Sanofi Pasteur and Actelion and holds research grants from CDC and US Dept of Veterans Affairs Research Service;

Dr. Saeed has no disclosures;

Dr. Mahé reports other from Sanofi Pasteur, outside the submitted work; Dr. Dubberke reports personal fees from Sanofi-Pasteur during the conduct of the study; grants from Microdermis, personal fees and other from Cubist, Merck, and Rebiotix outside the submitted work. 


\section{Authors' contributions}

$M O, Y Y-X, C K, D G, M C$, and ED conceived of and designed the study. MO, YY-X, DS, and MS had access to the data in the study and performed the analyses. MO drafted the manuscript, and all authors read and approved the final manuscript and agree to be held accountable for all aspects of the work.

\section{Acknowledgements}

We would like to thank L. Clifford McDonald, MD, Centers for Disease Control and Prevention for his advice and input. We also acknowledge access to data and services from the Washington University Center for Administrative Data Research, supported in part by grant UL1 TR000448 from the National Center for Advancing Translational Sciences (NCATS) of the National Institutes of Health $(\mathrm{NIH})$ and grant R24 HS19455 through the Agency for Healthcare Research and Quality (AHRQ).

\section{Funding}

The funding for this study was provided by Sanofi-Pasteur. The sponsor participated in study design, interpretation of data, and final review of the manuscript.

\section{Author details}

'Division of Infectious Diseases, Washington University School of Medicine, Campus Box 8051660 S. Euclid Ave, St. Louis 63110MO, USA. ${ }^{2}$ Department of Surgery, Washington University School of Medicine, St. Louis, MO, USA. ${ }^{3}$ Department of Psychiatry, Geisel School of Medicine at Dartmouth, Hanover, $\mathrm{NH}$, USA. ${ }^{4}$ Department of Medicine, Harvard Medical School, Boston, MA, USA. ${ }^{5}$ Edward Hines Jr. Veterans Affairs Hospital, Hines, IL, USA and Loyola University Chicago Stritch School of Medicine, Maywood, IL, USA.

${ }^{6}$ Sanofi-Pasteur, Lyon, France.

Received: 15 July 2015 Accepted: 9 April 2016

Published online: 22 April 2016

\section{References}

1. Lucado J, Gould C, Elixhauser A. Clostridium difficile Infection (CDI) Hospital Stays, 2009. HCUP Staistical Brief \#124. Agency for Healthcare Research and Quality, Rockville, MD 2012. http://www.hcup-us.ahrq.gov/reports/statbriefs/ sb124.pdf. Accessed 15 May 2015.

2. Lessa FC, Mu Y, Bamberg WM, Beldavs ZG, Dumyati GK, Dunn JR, et al. Burden of Clostridium difficile infection in the United States. N Engl J Med. 2015;372:825-34

3. Agency for Healthcare Research and Quality. HCUPnet, Healthcare Cost and Utilization Project. Agency for Healthcare Research and Quality 2015 http://hcupnet.ahrq.gov. Accessed 16 Feb 2015.

4. Hall AJ, Curns AT, MCDonald LC, Parashar UD, Lopman BA. The roles of Clostridium difficile and norovirus among gastroenteritis-associated deaths in the United States, 1999-2007. Clin Infect Dis. 2012;55:216-23.

5. Pepin J, Valiquette L, Cossette B. Mortality attributable to nosocomial Clostridium difficile-associated disease during an epidemic caused by a hypervirulent strain in Quebec. CMAJ. 2005;173:1037-42.

6. Dubberke ER, Butler AM, Reske KA, Agniel D, Olsen MA, D'Angelo G, et al. Attributable outcomes of endemic Clostridium difficile-associated disease in nonsurgical patients. Emerg Infect Dis. 2008;14:1031-8.

7. Kwon JH, Olsen MA, Dubberke ER. The morbidity, mortality, and costs associated with Clostridium difficile infection. Infect Dis Clin North Am. 2015;29:123-34.

8. Bateman BT, Rassen JA, Schneeweiss S, Bykov K, Franklin JM, Gagne JJ, et al. Adjuvant vancomycin for antibiotic prophylaxis and risk of Clostridium difficile infection after coronary artery bypass graft surgery. J Thorac Cardiovasc Surg. 2013;146:472-8.

9. Dial S, Delaney JA, Schneider V, Suissa S. Proton pump inhibitor use and risk of community-acquired Clostridium difficile-associated disease defined by prescription for oral vancomycin therapy. CMAJ. 2006;175:745-8.

10. Dubberke ER, Reske KA, Yan Y, Olsen MA, McDonald LC, Fraser VJ. Clostridium difficile-associated disease in a setting of endemicity: identification of novel risk factors. Clin Infect Dis. 2007:45:1543-9.

11. Gaynes R, Rimland D, Killum E, Lowery HK, Johnson TM, Killgore G, et al. Outbreak of Clostridium difficile infection in a long-term care facility: association with gatifloxacin use. Clin Infect Dis. 2004;38:640-5.
12. Muto CA, Pokrywka M, Shutt K, Mendelsohn AB, Nouri K, Posey K, et al. A large outbreak of Clostridium difficile-associated disease with an unexpected proportion of deaths and colectomies at a teaching hospital following increased fluoroquinolone use. Infect Control Hosp Epidemiol. 2005:26:273-80

13. Pepin J, Saheb N, Coulombe MA, Alary ME, Corriveau MP, Authier S, et al. Emergence of fluoroquinolones as the predominant risk factor for Clostridium difficile-associated diarrhea: a cohort study during an epidemic in Quebec. Clin Infect Dis. 2005:41:1254-60.

14. Zilberberg MD, Tabak YP, Sievert DM, Derby KG, Johannes RS, Sun X, et al. Using electronic health information to risk-stratify rates of Clostridium difficile infection in US hospitals. Infect Control Hosp Epidemiol. 2011;32: 649-55.

15. Loo VG, Poirier L, Miller MA, Oughton M, Libman MD, Michaud S, et al. A predominantly clonal multi-institutional outbreak of Clostridium difficileassociated diarrhea with high morbidity and mortality. N Engl J Med. 2005; 353:2442-9.

16. Chitnis AS, Holzbauer SM, Belflower RM, Winston LG, Bamberg WM, Lyons C et al. Epidemiology of community-associated Clostridium difficile infection, 2009 through 2011. JAMA Intern Med. 2013;173:1359-67.

17. Cohen SH, Gerding DN, Johnson S, Kelly CP, Loo VG, McDonald LC, et al. Clinical practice guidelines for Clostridium difficile infection in adults: 2010 update by the Society for Healthcare Epidemiology of America (SHEA) and the Infectious Diseases Society of America (IDSA). Infect Control Hosp Epidemiol. 2010;31:431-55.

18. MCDonald LC, Coignard B, Dubberke E, Song X, Horan T, Kutty PK Recommendations for surveillance of Clostridium difficile-associated disease. Infect Control Hosp Epidemiol. 2007;28:140-5.

19. Kuntz JL, Polgreen PM. The importance of considering different healthcare settings when estimating the burden of Clostridium difficile. Clin Infect Dis. 2015;60:831-6.

20. Dubberke ER, Butler AM, Yokoe DS, Mayer J, Hota B, Mangino JE, et al. Multicenter study of Clostridium difficile infection rates from 2000 to 2006. Infect Control Hosp Epidemiol. 2010;31:1030-7.

21. Schmiedeskamp M, Harpe S, Polk R, Oinonen M, Pakyz A. Use of international classification of diseases, ninth revision, clinical modification codes and medication use data to identify nosocomial Clostridium difficile infection. Infect Control Hosp Epidemiol. 2009;30:1070-6.

22. Dubberke ER, Reske KA, McDonald LC, Fraser VJ. ICD-9 codes and surveillance for Clostridium difficile-associated disease. Emerg Infect Dis. 2006;12:1576-9.

23. Dubberke ER, Butler AM, Nyazee HA, Reske KA, Yokoe DS, Mayer J, et al. The impact of ICD-9-CM code rank order on the estimated prevalence of Clostridium difficile infections. Clin Infect Dis. 2011:53:20-5.

24. Scheurer DB, Hicks LS, Cook EF, Schnipper JL. Accuracy of ICD-9 coding for Clostridium difficile infections: a retrospective cohort. Epidemiol Infect. 2007; 135:1010-3

25. Welker JA, Bertumen JB. Toxin assay is more reliable than ICD-9 data and less time-consuming than chart review for public reporting of Clostridium difficile hospital case rates. J Hosp Med. 2012;7:170-5.

26. Young-Xu Y, Kuntz JL, Gerding DN, Neily J, Mills P, Dubberke ER, et al. Clostridium difficile infection among veterans health administration patients. Infect Control Hosp Epidemiol. 2015;36:1038-45.

27. Lessa FC, Mu Y, Winston LG, Dumyati GK, Farley MM, Beldavs ZG, et al. Determinants of Clostridium difficile infection incidence across diverse United States geographic locations. Open Forum Infect Dis. 2014;1, ofu048.

28. Centers for Disease Control and Prevention (CDC). Vital signs: preventing Clostridium difficile infections. MMWR Morb Mortal Wkly Rep. 2012;61:157-62. 